\title{
The role of chorion on toxicity of silver nanoparticles in the embryonic zebrafish assay
}

\author{
Ki-Tae Kim ${ }^{1}$, Robert L. Tanguay ${ }^{2}$ \\ ${ }^{1}$ Department of Environmental Engineering, Seoul National University of Science and Technology, Seoul, Korea; ${ }^{2}$ Department \\ of Environmental and Molecular Toxicology, Oregon State University, Corvallis, OR, USA
}

\begin{abstract}
Objectives This study was designed to investigate how the size- and surface coating-dependent toxicity of silver nanoparticles (AgNPs) is influenced by the presence and absence of the chorion in an embryonic zebrafish assay.

Methods Normal and dechorinated embryos were exposed to four different AgNPs, 20 or $110 \mathrm{~nm}$ in size, with polypyrrolidone (PVP) or citrate surface coatings in a standard zebrafish embryo medium (EM). This was then compared to a $62.5 \mu \mathrm{M}$ calcium chloride $\left(\mathrm{CaCl}_{2}\right)$ solution where agglomeration was controlled.

Results Embryonic toxicity in the absence of the chorion was greater than in its presence. The smaller $20 \mathrm{~nm}$ AgNPs were more toxic than the larger $110 \mathrm{~nm}$ AgNPs, regardless of the chorion and test media. However, surface coating affected toxicity, since PVPcoated AgNPs were more toxic than citrate-coated AgNPs; this was strongly affected by the presence of the chorion in both $\mathrm{EM}$ and $\mathrm{CaCl}_{2}$.

Conclusions Our results demonstrate the permeability function of the chorion on the size- and surface coating-dependent toxicity of AgNPs. Thereafter, careful experiment should be conducted to assess nanoparticle toxicity in zebrafish embryos.
\end{abstract}

\author{
Correspondence: \\ Ki-Tae Kim, PhD \\ 232 Gongneung-ro, Nowon-gu, Seoul \\ 139-743, Korea \\ Tel: $+82-2-970-6642$ \\ Fax: +82-2-971-5776 \\ E-mail: ktkim@seoultech.ac.kr \\ Received: August 13, 2014 \\ Accepted: November 20, 2014 \\ Published online: December 10, 2014 \\ This article is available from: http://e-eht.org/
}

Keywords Chorion, Silver nanoparticles, Zebrafish

\section{Introduction}

Safety and regulatory concerns about silver nanoparticles (AgNPs) are growing due to their extensive use in consumer products and increasing potential of exposure to human and the environment [1]. According to the survey of Nanotechnology Consumer Products Inventory (http://www.nanotechproject. org), AgNPs are the most widely used engineered nanomaterials (ENMs). Approximately $25 \%$ of commercial products released onto the market contain AgNPs.

The embryonic zebrafish assay is an appealing in vivo model in (eco)toxicology [2]. Zebrafish embryos are optically transparent, allowing for easy observations of phenotypic responses in the internal organs including the brain, jaw, eye, heart, yolk sac, notochord, trunk, and tail. Furthermore, molecular techniques, both established and in development, can be applied to assess toxicity of chemicals. More importantly, toxicological outcomes obtained from zebrafish may be extrapolated to human biology due to their homology and relevance to the physiological responses of mammals $[3,4]$.

The embryonic zebrafish provides considerable experimental flexibility and versatility in nanotoxicology [5]. To investigate toxic mode of action of AgNPs, in this study, we used zebrafish embryos that had developed normally and exposed them for 5 days in a test medium of low ionic strength where agglomeration was excluded [6]. We hypothesized that an attempt to control NP agglomeration (i.e., monodispersion of AgNPs) in the test media would enable us to determine the size- and surface coating-dependent toxicity of AgNPs accurately and reliably. In this study, we exposed dechorionated embryos to four different types of AgNPs to minimize chorion interference, because the chorion plays a role in protecting the embryo from external in- 
fluences, including chemical exposure. Although the removal of the chorion is helpful in elucidating the mechanisms of toxicity of ENMs, questions concerning interference of the chorion in AgNP toxicity remain, because ecological toxicity testing has, in general, been performed in the presence of the chorion.

Although no comprehensive investigation of chorion permeability or sensitivity to chemicals has yet been reported, the chorion is suspected to be a barrier to the entry of NPs into zebrafish embryos $[7,8]$. The chorion possesses canals, the pore size of which is approximately 0.6-0.7 $\mu \mathrm{m}$, larger than the size of the NP. Lee et al. [7] first visualized AgNP penetration through chorion canals. However, although the pore size is known to be larger than the NP size, in particular, the effect of the chorion on NP transport and subsequent biological toxicity may be complicated when NPs are agglomerated. The presence of the chorion is a critical concern on varied developmental toxicity of zebrafish embryos exposed to various formulations of AgNPs to understand the effect of size and surface coating [3,9]. Consequently, the systematic examination of the changes in the biological responses of zebrafish embryos exposed to NPs in the presence or absence of the chorion is necessary.

The objective of this study was to investigate how the size- and surface coating-dependent toxicity of AgNPs varied according to the presence or absence of the chorion in an embryonic zebrafish assay. We used 20 and $110 \mathrm{~nm}$ sized AgNPs, and AgNPs were coated with either polypyrrolidone (PVP) or citrate. The citrate and PVP are the most widespread and most commonly used stabilizer in AgNP synthesis. In order to determine the permeability differential of the AgNPs, we exposed normal and dechorionated embryos to four different AgNPs in a standard zebrafish embryo medium (EM) and $62.5 \mu \mathrm{M}$ calcium chloride $\left(\mathrm{CaCl}_{2}\right)$ solution. We observed that AgNPs were monodispersed in $\mathrm{CaCl}_{2}$ without agglomeration, and the exposure of zebrafish embryos in $\mathrm{CaCl}_{2}$ to AgNPs was useful for the observation of the variations in biological toxicity in the presence and absence of the chorion. This can be compared further to EM in which AgNPs were agglomerated.

\section{Materials and Methods}

\section{Silver Nanoparticles and Characterization in Test Media}

AgNPs synthesized by NanoComposix (San Diego, CA, USA) were used. The AgNPs were $20 \mathrm{~nm}$ PVP-coated AgNPs (20AgNPs-P), $20 \mathrm{~nm}$ citrate-coated AgNPs (20AgNPs-C), $110 \mathrm{~nm}$ PVP-coated AgNPs (110AgNPs-P) and $110 \mathrm{~nm}$ citrate-coated AgNPs (110AgNPs-C). This set of AgNPs was one of the ENMs selected by the Nanotechnology Health Implications Re- search Consortium, an interdisciplinary program of the National Institute of Environmental Health Sciences, which addresses the health and safety concerns of AgNPs. We followed the guidelines for sample handling and storage recommended by the Nanotechnology Characterization Laboratory (NCL).

The NCL provided the physicochemical properties of AgNPs at a concentration of $100 \mathrm{mg} / \mathrm{L}$ suspended with $2 \mathrm{mM} \mathrm{NaCl}$. The z-average and zeta potentials of four AgNPs were measured using dynamic light scattering (Malvern Zetasizer Nano ZS Instrument, Southborough, MA, USA) with a back scattering detector $\left(173^{\circ}\right)$. The core diameter was measured by transmission electron microscopy, also provided by the NCL (NCL-NIEHS201111A). In this study, we analyzed and compared the z-average of AgNPs in EM and $\mathrm{CaCl}_{2}$ by using a ZetaPALS (Brookhaven Instruments, Holtsville, NY, USA) after the preparation of 10 -fold diluted aliquot from $1,000 \mathrm{mg} / \mathrm{L}$ of stock solution.

\section{Optimization of 62.5 $\mu \mathrm{M}$ Calcium Chloride}

Because zebrafish embryos can develop normally in low ionic strength media [10], we tested media of various ionic strengths and found that incubation of embryos in $1 \mathrm{mM} \mathrm{CaCl}_{2}$ did not induce developmental morbidity or mortality at 120 hours post fertilization (hpf) [6]. To ascertain the minimum concentration of $\mathrm{CaCl}_{2}$, we carried out a titration, and determined that 62.5 $\mu \mathrm{M} \mathrm{CaCl}_{2}$ was low sufficient for normal development until 120 hpf and nonagglomeration of AgNPs.

\section{Zebrafish Maintenance and Embryonic Zebrafish Assay}

Adult zebrafish (Danio rerio) were raised at Oregon State University's Sinnhuber Aquatic Research Laboratory in a water flow-through system that maintained a constant temperature of $28^{\circ} \mathrm{C}$ under a 14:10 hours light-dark photoperiod. Embryos collected and staged [11] were from the tropical 5D zebrafish line and were cultured in buffered $\mathrm{EM}(\mathrm{pH} 7 \pm 0.2)$ using reverse osmosis water. The standard EM consisted of $15 \mathrm{mM} \mathrm{NaCl}, 0.5$ $\mathrm{mM} \mathrm{KCl}_{1} 1 \mathrm{mM} \mathrm{CaCl} 2,1 \mathrm{mM} \mathrm{MgSO}_{4}, 0.15 \mathrm{mM} \mathrm{KH}_{2} \mathrm{PO}_{4}, 0.05$ $\mathrm{mMNa}_{2} \mathrm{HPO}_{4}$ and $0.7 \mathrm{mMNaHCO}_{3}[12]$.

Healthy staged embryos were loaded into 96-well plates filled with $90 \mu \mathrm{L}$ of test medium and $10 \mu \mathrm{L}$ of the AgNP suspension. To achieve the final concentrations of $0.8,4,20,10$, and $50 \mathrm{mg} /$ $\mathrm{L}$, after a $10 \times$ concentrated, serial diluted concentration of AgNPs was prepared, $10 \mu \mathrm{L}$ of the AgNP suspension was mixed into the respective wells. Exposure to AgNPs was initialized at 6 hpf. Eight embryos were exposed to each treatment with four replicates. We evaluated a total of 22 endpoints including mor- 
tality and malformation at 24 and $120 \mathrm{hpf}$. The percentage of malformation was calculated by the number of embryos affected per living embryos at each treatment. Screened malformations were: yolk sac edema; axis, eye, snout, jaw, and otic (ear) defects; pericardial edema; brain, somite, and pectoral and caudal fin defects; pigmentation abnormality; circulation and trunk defects; swim bladder abnormality; notochord defects, and touch response abnormality.

Using pronase (Sigma-Aldrich, St Louis, MO, USA), we removed the chorion in an automated dechorinator at $4 \mathrm{hpf}$. Briefly, after introducing pronase, the plate was shaken for 5 minutes, washed and then the shaking procedure was repeated. While dechorionated embryos were placed into each well using an automated embryo placement systems, normal embryos (i.e., undechorionated embryos) were transferred into a 96-well plate by hand. The ability to produce healthy embryos was confirmed in ae previous study [13]. No detectable mortality or morbidity of dechorionated embryos in the control group was observed over 5 days in this study.

\section{Results}

\section{Characterization of Silver Nanoparticles}

We determined the z-average of each AgNPs in EM and $\mathrm{CaCl}_{2}$, and compared them to those provided by the NCL (Fig-

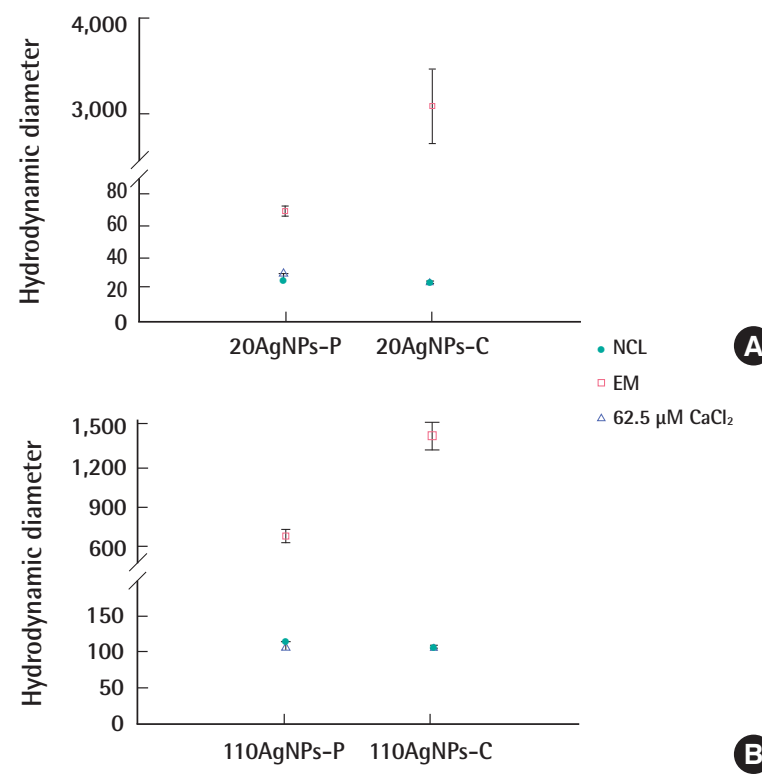

Figure 1. The z-average of four silver nanoparticles (AgNPs) suspended in (A) embryo medium (EM) and (B) 62.5 uM calcium chloride (CaCl2). Error bars represent standard deviations. NCL, Nanotechnology Characterization Laboratory; 20AgNPs-P, 20 nm polypyrrolidone (PVP)-coated AgNPs; 20AgNPs-C, 20 nm citrate-coated AgNPs; 110AgNPs-P, 110 nm PVPcoated AgNPs; 110 AgNPs-C, $110 \mathrm{~nm}$ citrate-coated AgNPs. ure 1). We noted that the stock solution of $1,000 \mathrm{mg} / \mathrm{L}$ AgNPs provided by NanoComposix was suspended in $2 \mathrm{mM} \mathrm{NaCl}$, where the NCL measured the reference of the z-average. Depending on the AgNPs, the z-average increased dramatically up to micro-size in EM. The z-average of 20AgNPs-P, 20AgNPs-C, 110 AgNPs-P, and 110AgNPs-C increased to $69.7 \pm 2.7,3078.4$ $\pm 400.7,691.8 \pm 40.3$, and $1400.5 \pm 91.2 \mathrm{~nm}$ (mean \pm standard deviation), respectively. Although it appeared that the citrate surface coating caused more agglomeration, it is not clear why the diameter of the 20AgNPs- $\mathrm{C}$ was larger than that of the 110AgNPs-C. Further, the z-average of AgNPs in $\mathrm{CaCl}_{2}$ were totally different from those in EM. All AgNPs were stably dispersed (i.e., no increas in z-average), the z-average measured did not differ significantly from those provided by the NCL (Figure 1). The z-average of 20AgNPs-P, 20AgNPs-C, 110 AgNPs-P, and $110 \mathrm{AgNPs}-\mathrm{C}$ were determined to be $30.5 \pm 1.2,26.1 \pm 0.8$, $123.2 \pm 0.9$, and $100.4 \pm 2.1 \mathrm{~nm}$, respectively. The suspension of 20AgNPs and 110AgNPs exhibited a bright and slightly darker yellow color, respectively, but their color turned to black when transferred into EM, indicating agglomeration, whereas the color did not change in $\mathrm{CaCl}_{2}$. These z-average corroborate $\mathrm{UV}$-vis spectra analysis (data not shown). The absorbance of AgNPs was maintained over 5 days in $\mathrm{CaCl}_{2}$ while a drop in absorbance was observed in EM. After 5 days, the absorbance of 20AgNPs$\mathrm{P}$ was attenuated and that of 20AgNPs-C, 110AgNPs-P, and 110AgNPs-C disappeared completely in EM, indicating NP agglomeration. The NCL report stated that all AgNPs exhibited negative zeta-potentials (NCL-NIEHS201111A). Because there is no consistent correlation between zeta-potential and either z-average or $\mathrm{UV}$-vis absorbance spectra in $2 \mathrm{mM} \mathrm{NaCl}$ where agglomeration is controlled, zeta-potentials were not measured in EM and $\mathrm{CaCl}_{2}$ used in this study. The electrostatic repulsion might not be dominant in maintaining AgNP stability in suspension in comparison with the interfacial forces [14]. Taken together, Ag$\mathrm{NPs}$ were dispersed stably in $\mathrm{CaCl}_{2}$. We hypothesized that the exposure of nonagglomerated AgNPs helped us to investigate the effect of the chorion on AgNPs with variable size and surface coating.

\section{Embryonic Toxicity of Silver Nanoparticles}

The Figures 2 and 3 show the total percentage of mortality and malformation of embryos exposed to four AgNPs in the presence and absence of the chorion in $\mathrm{EM}$ and $\mathrm{CaCl}_{2}$. A total of 18 endpoints evaluated at $120 \mathrm{hpf}$ was used for the toxicity differential of each treatment. The black color indicates the mortality at $120 \mathrm{hpf}$ over the range of AgNP exposure concentrations $(0,0.08,0.4,2,1,0$ and $50 \mathrm{mg} / \mathrm{L})$. The percent inci- 

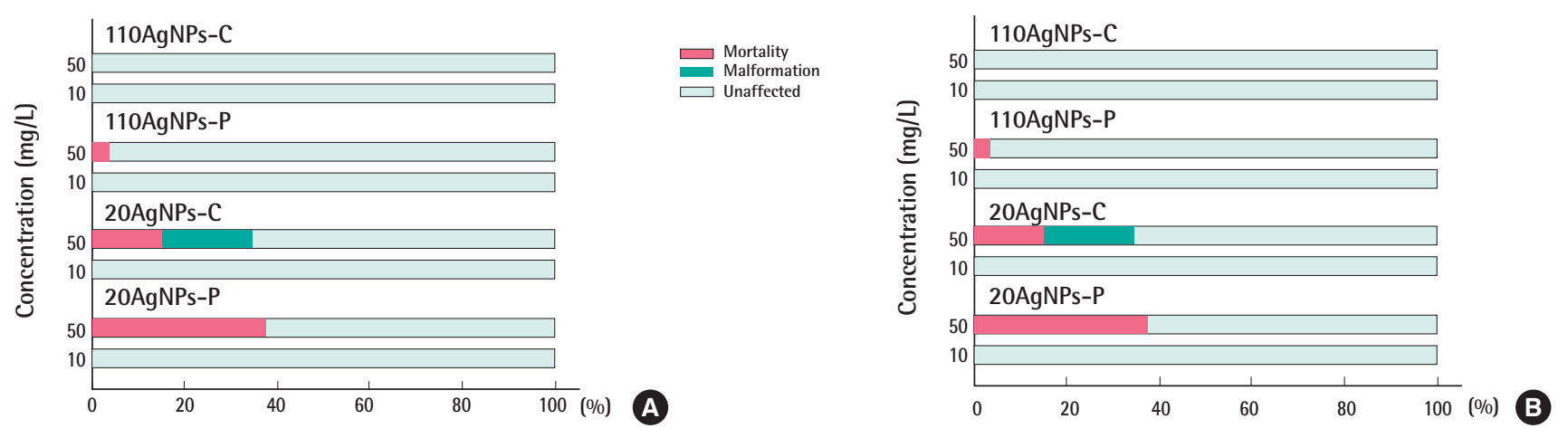

Figure 2. Embryonic toxicity of four silver nanoparticles (AgNPs) suspended in (A) embryo medium and (B) 62.5 uM calcium chloride in the presence of the chorion. Eight embryos were exposed and four replicates were used for each treatment. No detectable mortality and malformation were observed at 0, 0.08, 0.4, 2 mg/L at each treatment. 110AgNPs-C, $110 \mathrm{~nm}$ citrate-coated AgNPs; 110AgNPs-P, $110 \mathrm{~nm}$ polypyrrolidone (PVP)-coated AgNPs; 20AgNPs-C, 20 nm citrate-coated AgNPs; 20AgNPs-P, 20 nm PVP-coated AgNPs.
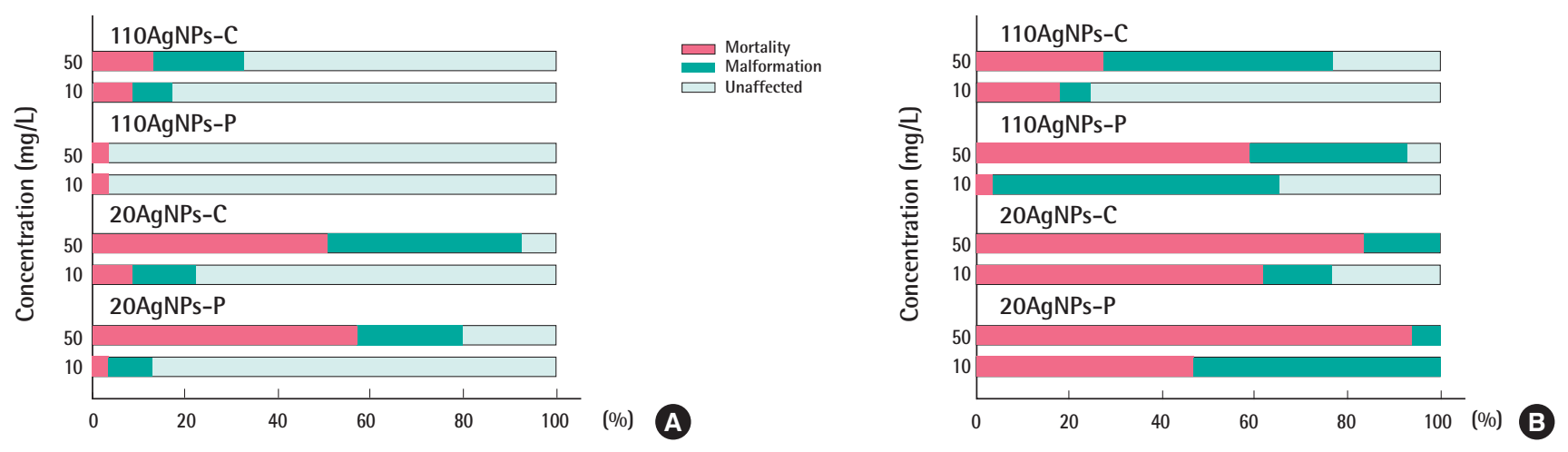

Figure 3. Embryonic toxicity of four silver nanoparticles (AgNPs) suspended in (A) embryo medium and (B) 62.5 uM calcium chloride in the absence of the chorion. Eight embryos were exposed and four replicates were used for each treatment. No detectable mortality or malformation was observed at 0, 0.08, 0.4, 2 mg/L for each treatment. 110AgNPs-C, $110 \mathrm{~nm}$ citrate-coated AgNPs; 110AgNPs-P, $110 \mathrm{~nm}$ polypyrrolidone (PVP)-coated AgNPs; 20AgNPs-C, 20 nm citrate-coated AgNPs; 20AgNPs-P, 20 nm PVP-coated AgNPs.

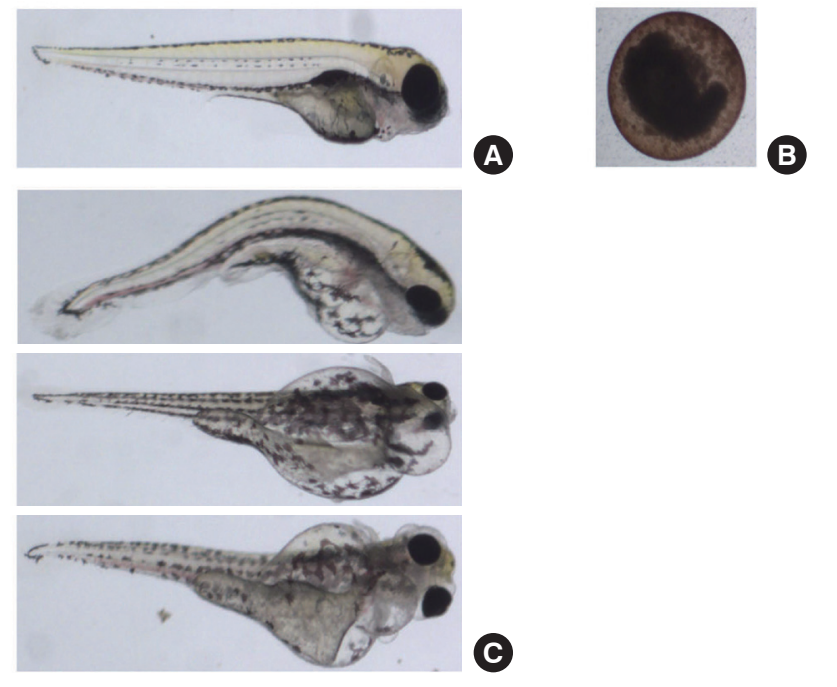

Figure 4. Representative images of embryos exposed to four silver nanoparticles (AgNPs) at 120 hpf: (A) control, (B) unhatched embryos, mainly observed at $50 \mathrm{mg} / \mathrm{L}$ of polypyrrolidone (PVP)-coated AgNPs (AgNPs-P) and citrate-coated AgNPs (AgNPs-C) in the presence of the chorion, (C) malformed embryos. dence of malformation indicates the percentage of surviving embryos that exhibited any of the 17 morphological malformations described above. Embryonic toxicity to AgNPs suspended in EM was significantly lower than those suspended in $\mathrm{CaCl}_{2}$, probably due to the low bioavailability that resulted from strong agglomeration, which was expected from the increase in the $\mathrm{z}$ average and the disappearance of UV-vis absorbance in EM. A previous study showed that non-agglomerated AgNPs accumulated to a greater degree in embryos [6]. Typically, embryonic toxicity in the absence of the chorion was higher than in the presence of the chorion. Embryos that were counted as malformed in the presence of the chorion showed only one malformation, for example, mainly yolk sac edema. This indicates that the chorion definitely serves as a barrier to AgNP exposure and contact with the embryos. A quantitative study of the uptake of AgNPs according to the presence and absence of the chorion is needed.

Figure 4 illustrates examples of embryos exposed to each Ag- 
NPs for 24 and $120 \mathrm{hpf}$. The greatest embryonic toxicity was observed at the highest concentration of $50 \mathrm{mg} / \mathrm{L}$ in both test media, regardless of the presence of the chorion. Below concentrations of $50 \mathrm{mg} / \mathrm{L}$, a very low incidence of mortality and malformation was observed. In the presence of the chorion, embryonic death or decay without hatching was typical type of mortality observed at $50 \mathrm{mg} / \mathrm{L}$. Below concentrations of $50 \mathrm{mg} / \mathrm{L}$, no malformations were caused by any of the AgNPs in the hatched larvae. In the absence of the chorion, 20AgNPs-P and 20AgNPs-C caused some mortality at $10 \mathrm{mg} / \mathrm{L}$, and approximately $70 \%$ of the embryos showed malformation of the yolk sac edema in 110AgNPs-P at $10 \mathrm{mg} / \mathrm{L}$. Malformations observed in the absence of the chorion were jaw and eye abnormalities, pericardial and yolk sac edema, and snout and circulation defects.

\section{Discussion}

Based on the percentage of mortality and malformation, we ranked the embryonic toxicity of AgNPs in EM in the presence of the chorion as: 20 AgNPs-P $>20$ AgNPs-C $>110$ AgNPs-C $\approx$ 110 AgNPs-P and in the absence of the chorion as 20AgNPs$\mathrm{C}>20$ AgNPs-P $>110$ AgNPs-C $>110$ AgNPs-P. The incidence of mortality was considered to be more toxic than malformation. The presence of the chorion changed the order of toxicity with respect to the surface coating rather than the size. It is not clear, however, that 20AgNPs-C with a larger z-average in EM were more toxic than $110 \mathrm{~nm} \mathrm{AgNPs}$, regardless of the presence of the chorion. In $\mathrm{CaCl}_{2}$, the embryonic toxicity of AgNPs was ranked as 20AgNPs-P $>20$ AgNPs-C $>110$ AgNPs-P $\approx 110 \mathrm{Ag}$ NPs-C and 20AgNPs-P $>20 \mathrm{AgNPs}-\mathrm{C}>110 \mathrm{AgNPs}-$ $\mathrm{P}>110 \mathrm{AgNPs}-\mathrm{C}$ in the presence and absence of the chorion, respectively. We noted that in the environment in which particle agglomeration (i.e., in $\mathrm{CaCl}_{2}$ ) and chorion interference (i.e., in the absence of the chorion) were controlled, PVP was more toxic than the citrate coating, regardless of the particle size, as shown above. In $\mathrm{CaCl}_{2}$, where agglomeration was controlled, a change in the order of toxicity also occurred, predominantly regarding the surface coating in the presence of the chorion. Consequently, the presence of the chorion did influence the interaction with the chemical surface coating material, rather than the physical size effect in the embryonic toxicity of AgNPs, regardless of the test medium. A further study comparing the effect of chorion on AgNPs with sizes between 20 and $110 \mathrm{~nm} \mathrm{AgNPs} \mathrm{is}$ needed.

To our knowledge, this is the first report of a systematic comparison of ENM toxicity with respect to chorion permeability. As demonstrated, the size- and surface coating-dependent toxic- ity of AgNPs was controlled by the function of embryonic permeability. Embryonic permeability was affected by particle agglomeration, and primarily caused a differential in toxicity dependent on the surface coating. The embryonic zebrafish assay is a well-established model not only for rapid and cost-efficient toxicity screening $[15,16]$, but also for the identification of molecular mechanisms [17] in assessing ENM toxicity. However, knlowedge with regard to the permeability limitations and secondary phenotypic responses to the chorion related to the hatching process in the presence of the chorion [13] is lacking in nanotoxicology. Therefore, further experimental investigations of the effect of the chorion should be carefully considered in assessing the toxicity of ENMs and other chemicals in the embryonic zebrafish assay.

\section{Acknowledgements}

This study was supported by the Research Program funded by Seoul National University of Science and Technology.

\section{Conflict of Interest}

The authors have no conflicts of interest with material presented in this paper.

\section{References}

1. US Environmental Protection Agency. State of the science literature review: everything nanosilver and more; 2010 [cited 2014 Aug 10]. Available from: http://www.epa.gov/nanoscience/files/ NanoPaper1.pdf.

2. Hill AJ, Teraoka H, Heideman W, Peterson RE. Zebrafish as a model vertebrate for investigating chemical toxicity. Toxicol Sci 2005;86(1):6-19.

3. Parng C. In vivo zebrafish assays for toxicity testing. Curr Opin Drug Discov Devel 2005;8(1):100-106.

4. Howe K, Clark MD, Torroja CF, Torrance J, Berthelot C, Muffato $\mathrm{M}$, et al. The zebrafish reference genome sequence and its relationship to the human genome. Nature 2013;496(7446):498-503.

5. Kim KT, Tanguay RL. Integrating zebrafish toxicology and nanoscience for safer product development. Green Chem 2013;15(4):872880.

6. Kim KT, Truong L, Wehmas L, Tanguay RL. Silver nanoparticle toxicity in the embryonic zebrafish is governed by particle dispersion and ionic environment. Nanotechnology 2013;24(11):115101.

7. Lee KJ, Nallathamby PD, Browning LM, Osgood CJ, Xu XH. In vivo imaging of transport and biocompatibility of single silver nanoparticles in early development of zebrafish embryos. ACS Nano 2007;1(2):133-143.

8. Duan J, Yu Y, Shi H, Tian L, Guo C, Huang P, et al. Toxic effects of silica nanoparticles on zebrafish embryos and larvae. PLoS One 
2013;8(9):e74606.

9. Powers CM, Slotkin TA, Seidler FJ, Badireddy AR, Padilla S. Silver nanoparticles alter zebrafish development and larval behavior: distinct roles for particle size, coating and composition. Neurotoxicol Teratol 2011;33(6):708-714.

10. Truong L, Zaikova T, Richman EK, Hutchison JE, Tanguay RL. Media ionic strength impacts embryonic responses to engineered nanoparticle exposure. Nanotoxicology 2012;6(7):691-699.

11. Kimmel CB, Ballard WW, Kimmel SR, Ullmann B, Schilling TF. Stages of embryonic development of the zebrafish. Dev Dyn 1995;203(3):253-310.

12. Westerfield $M$. The zebrafish book: a guide for the laboratory use of zebrafish (Danio rerio). 4th ed. Oregon: University of Oregon Press; 2000.

13. Mandrell D, Truong L, Jephson C, Sarker MR, Moore A, Lang C, et al. Automated zebrafish chorion removal and single embryo placement: optimizing throughput of zebrafish developmental tox- icity screens. J Lab Autom 2012;17(1):66-74.

14. Jin X, Li M, Wang J, Marambio-Jones C, Peng F, Huang X, et al. High-throughput screening of silver nanoparticle stability and bacterial inactivation in aquatic media: influence of specific ions. Environ Sci Technol 2010;44(19):7321-7328.

15. George S, Xia T, Rallo R, Zhao Y, Ji Z, Lin S, et al. Use of a highthroughput screening approach coupled with in vivo zebrafish embryo screening to develop hazard ranking for engineered nanomaterials. ACS Nano 2011;5(3):1805-1817.

16. Lin S, Zhao Y, Ji Z, Ear J, Chang CH, Zhang H, et al. Zebrafish high-throughput screening to study the impact of dissolvable metal oxide nanoparticles on the hatching enzyme, ZHE1. Small 2013;9(9-10):1776-1785.

17. Kim KT, Zaikova T, Hutchison JE, Tanguay RL. Gold nanoparticles disrupt zebrafish eye development and pigmentation. Toxicol Sci 2013;133(2):275-288. 